\section{Peter Schnider \\ Ecaterina Moraru \\ Marion Vigl \\ Christian Wöber \\ Daniela Földy \\ Joachim Maly \\ Christian Bittner \\ Peter Wessely \\ Eduard Auff}

Received: 12 July 2001

Accepted in revised form: 1 July 2002

P. Schnider $(\bowtie) \cdot$ E. Moraru • M. Vigl

C. Wöber $\bullet$ D. Földy • J. Maly $・$ P. Wessely

E. Auff

Department of Neurology,

Faculty of Medicine,

University of Vienna,

Währinger Gürtel 18-20,

A-1090 Vienna, Austria

e-mail: peter.schnider@univie.ac.at

Tel.: +43-1-40400-3120

Fax: +43-1-40400-31241

\section{Bittner}

Department of Physical Medicine and Rehabilitation,

Faculty of Medicine,

University of Vienna, Vienna, Austria

\title{
Physical therapy and adjunctive botulinum toxin type $A$ in the treatment of cervical headache: a double-blind, randomised, placebo-controlled study
}

\begin{abstract}
We examined the efficacy of physical therapy and adjunctive botulinum toxin type A (BTX-A) injections in the treatment of cervical headache. We performed a doubleblind, randomised, placebo-controlled study over a 12-week period in a university clinic outpatients department. A total of 33 patients with cervical headache, diagnosed according to International Headache Society classification were enrolled. All patients received standardized physical therapy over a three-week period. Patients were randomised to receive either BTX-A (Botox) or placebo. The BTX-A group received a total dose of 90 mouse units $(\mathrm{mu})$ BTX-A at six trigger points while the placebo group received saline. Pain characteristics were reported in a
\end{abstract}

headache diary. Tenderness in the neck muscles, the sagittal range of motion and biofeedback measurement were also documented. Both groups showed significant improvement in terms of headache severity $(p<0.05)$, number of headache-free days $(p=0.005)$ and number of headache hours per day $(p<0.05)$. Trends towards an increase in the number of headache-free days and a decrease in headache hours per day were observed in the BTX-A group. No major side effects were observed. Physical measures and BTX-A injections are safe and effective in the treatment of cervical headache.

Key words Cervical headache • Botulinum toxin type A - Physical therapy

\section{Introduction}

Botulinum toxin type A (BTX-A) represents an established form of treatment for focal dystonic syndromes [1]. Apart from improvement in the involuntary movement disorder, many patients describe an excellent analgesic effect after being treated with BTX-A. Consequently, pain disorders of muscular origin such as myofascial pain syndromes have been successfully treated with BTX-A by injecting it at the so-called trigger points [2, 3]. Recently, vehicle-controlled studies have investigated whether BTX-A injections are an effective treatment for various headache syndromes in which a myofascial component contributes to the genesis of pain [4-9].
Cervical headaches $(\mathrm{CH})$ are headaches due to a disorder in the neck which is usually associated with movement abnormalities [10]. The disorder may be located in the joints, ligaments, muscles or other soft tissues of the neck. Pain is activated by any form of stretching of the deeper musculoskeletal structures in the cervical spine, and conducted by deep, unmyelinated C-fibres [11, 12].

The prevalence and incidence of $\mathrm{CH}$ remains to be determined and depends on the diagnostic criteria used [13, 14]. The classification of $\mathrm{CH}$ is still an issue of debate. Whereas the term "cervicogenic headache" basically defines a unilateral headache without sideshift and a characteristic mechanical precipitation mechanism [15], the International Headache Society (IHS) has defined the enti- 
ty as uni- or bilateral pain localised in the neck and occipital region with projection to the forehead, orbital region, temples, vertex or ears [10]. The diagnostic criteria according to the IHS for $\mathrm{CH}$ are listed in Table 1. The differential diagnosis remains problematic as there is considerable overlap with other common headache forms, especially tension-type headache. However in other common types of headache, the proposed radiological abnormalities in combination with an obligatory pain pattern precipitated or aggravated by particular neck movements cannot be found so frequently.

BTX-A develops analgesic properties mainly by means of a muscle-relaxing effect accompanied by local changes in pain afferents and blood perfusion. As various types of muscle relaxation are often used to treat $\mathrm{CH}$ [16], BTX-A might be a useful adjunctive agent for treating head and neck pain caused by the contraction of neck muscles. In addition, it appears that BTX-A has a direct antinociceptive effect. It is hypothesized that BTX-A reduces the release of nociceptive neuropeptides from either cholinergic neurons or from $\mathrm{C}$ or A delta fibres [17].

Physical therapy is used as a standard treatment in patients with cervicogenic headache. It has been shown that physical therapy, i.e. mud packs, influence cytokine serum levels and hence contribute to pain relief by reducing inflammatory reactions [18]. In our study we examined whether the combined use of physical therapy, such as massages and hot mud packs, and intramuscular injections of BTX-A is more effective than physical therapy and placebo injections in the treatment of $\mathrm{CH}$.

Table 1 International Headache Society (IHS) classification (group 11): headache or facial pain associated with disorder of the cranium, neck, eyes, ears, nose, sinuses, teeth, mouth or other facial or cranial structures

Group 11. 2. 1 Cervical spine

\section{Diagnostic criteria}

A. Pain localized to neck and occipital region. May project to forehead, orbital region, temples, vertex or ears

B. Pain is precipitated or aggravated by particular neck movements or sustained neck posture

C. At least one of the following:

a. Resistance to or limitation of passive neck movements

b. Changes in neck muscle contour, texture, tone or response to active and passive stretching and contraction

c. Abnormal tenderness of neck muscles

D. Radiological examination reveals at least one of the following:

a. Movement abnormalities in flexion/extension

b. Abnormal posture

c. Fractures, congenital abnormalities, bone tumours, rheumatoid arthritis or other distinct pathology (not pondylosis or osteochondrosis)

\section{Patients and methods}

\section{Study design}

We performed a prospective, double-blind, randomised, placebocontrolled study on the efficacy and safety of BTX-A injections and physical therapy in comparison with placebo injections and physical therapy. The study lasted 16 weeks with a baseline period of 4 weeks (weeks 1-4) prior to BTX-A injection and a follow-up period of 12 weeks (weeks 5-16). Follow-up examinations were carried out 4,8 and 12 weeks after the injections with BTX-A or placebo.

Patients

A total of 33 patients ( 20 women) aged between 26 and 75 years (mean 50.7 years; SD, 10.4) with $\mathrm{CH}$ diagnosed according to the IHS classification [10] were included in this study. The inclusion criteria were a diagnosis of $\mathrm{CH}$ for at least 6 months, age of over 19 years, an inadequate or missing response to previous treatment and the presence of painful trigger and/or tender points. Headaches had to be present on more than 15 days per month. Exclusion criteria were other symptomatic headache forms, excessive consumption of analgesics as defined by the IHS, and pregnancy. The duration of headache was 0.5 to 30 years (mean 6 years; SD, 6.6). 16 patients had strictly unilateral headache without side shift, 9 had bilateral headache with marked unilateral dominance and 8 had bilateral headache. Three patients with a history of excessive analgesic intake and were included in the study 4 months after inpatient withdrawal-therapy. The following analgesics were taken by the majority of patients: salicylates, para-aminophenol derivates, antirheumatics, muscle relaxants. Four patients had an additional diagnosis of migraine, but each of them was able to distinguish between the two types of headache.

Randomisation and treatment

Patients were randomised into two groups. Patients of the treatment group received a total of 90 mouse units (mu) botulinum toxin type A (Botox, Allergen Inc., USA) in $0.9 \mathrm{ml}$ normal saline from a vial of $100 \mathrm{mu}$ reconstituted and diluted in $1 \mathrm{ml}$ saline. The placebo group received $0.9 \mathrm{ml}$ normal saline. A dose of $15 \mathrm{mu}$ Botox or $0.15 \mathrm{ml}$ saline was injected at each of the 6 most painful tender or trigger points of the cervical muscles. Both groups also received standardized physical therapy (massage and hot packs) for nine sessions (weeks 6-8).

Seventeen patients (7 men; mean age 51.4 years, SD 12.2; mean headache duration 6.1 years, SD 7.2) received Botox injections. Sixteen ( 6 men; mean age 50 years; SD 8.8; mean duration 6.1 years, SD 7.2) received placebo injections. One patient (placebo) refused to come for further control visits and was thus excluded from further analysis. There were no statistically significant differences between the two groups regarding age, gender or duration of headache. The study protocol was approved by the Ethics Committee of the University of Vienna and written informed consent was obtained from all patients. 


\section{Evaluation}

Patients kept a headache diary for the baseline and follow-up periods. The following items were documented: presence of headache (hours per day), number of headache-free days per 4-week period, number of analgesics per day, and the daily pain intensity using a visual analogue scale (VAS; $0=$ no pain, $100=$ worst pain).

The sagittal range of motion was documented as the difference (chin-jugulum distance) between maximum anteflexion and retroflexion in centimetres. The six most painful tender or trigger points of the neck and shoulder muscles were scored on a fourpoint scale (1, no local tenderness to pressure; 2 , moderate local tenderness to pressure; 3 , pronounced local tenderness to pressure; 4 , pronounced local tenderness to pressure with pain radiation).

Biofeedback measurements were performed at baseline and at the end of the study. Surface electrodes were placed on the trapezius muscle (superior and inferior part) and on the frontal belly of the occipitofrontalis muscle on both sides.

Side effects and serious adverse events were reported by the patients and graded by the investigator with respect to severity and relation to the treatment.

\section{Statistical analysis}

All data were analysed using the SAS General Linear models procedure analysis of variables (ANOVA). The number of patients who needed to be treated with BTX-A for a $>50 \%$ reduction in the number of headache-free days and headache hours per day was calculated for weeks $12-16$.

\section{Results}

At baseline (weeks 1-4), VAS (headache severity, graded by the patients), headache-free days, headache hours per day and analgesics intake per day did not differ significantly between groups, although there was a trend towards less headache-free days and more headache hours per day in the treatment group (Figs. 1-4).

During the follow-up period VAS showed a significant improvement in both groups $(p<0.05)$. There was no significant difference between them (Fig. 1).

There was a significant increase in the number of headache-free days in both groups with time $(p=0.005)$. There was a trend towards more headache-free days in the treatment group compared to the placebo group in weeks 13-16 (p<0.073) (Fig. 2).

The number of headache hours per day was significantly reduced in both groups during the follow-up period $(p<0.05)$. There was no significant difference between the two groups, but in weeks 5-8 a mild trend towards a greater

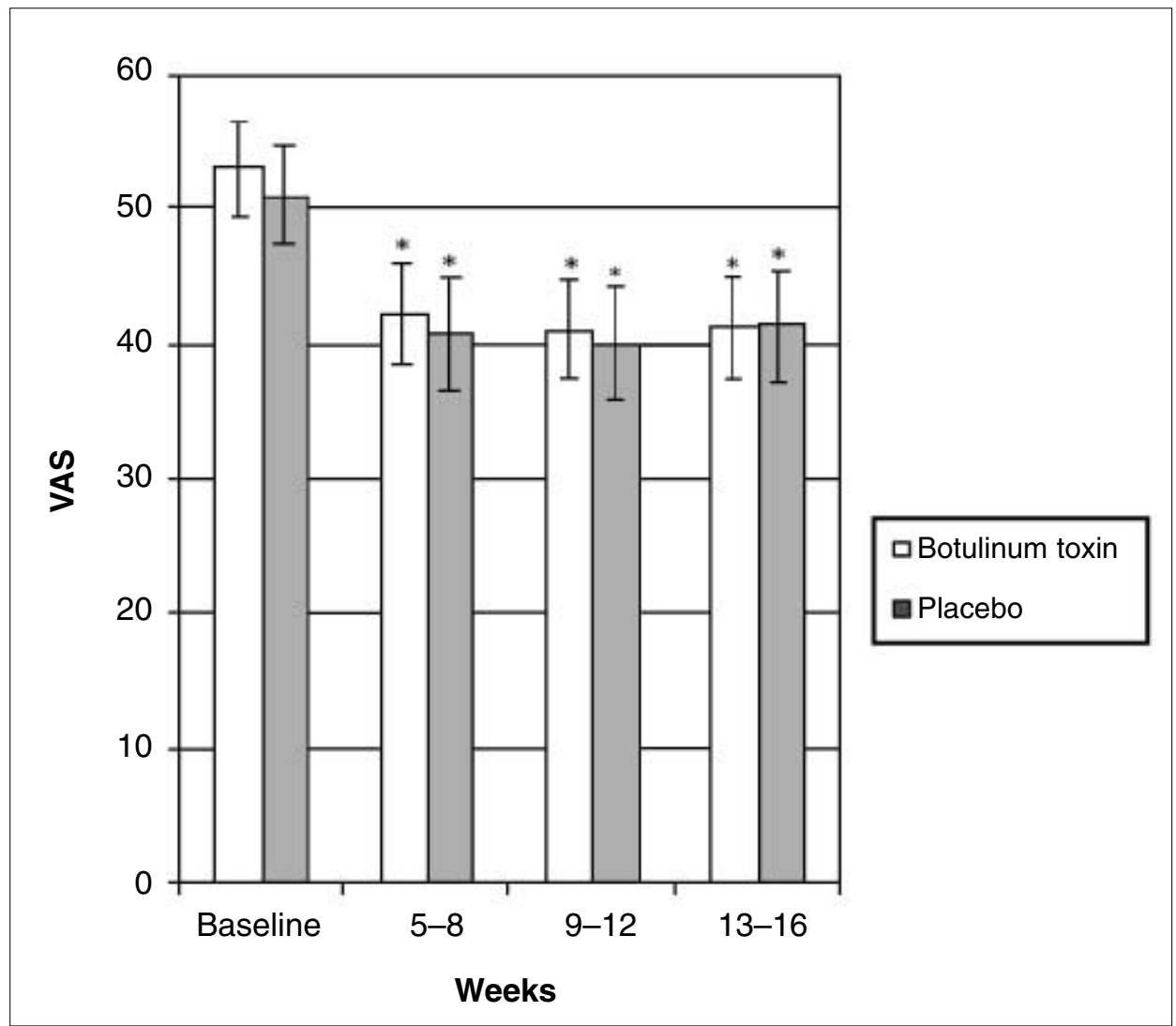

Fig. 1 Headache severity as scored on a visual analogue scale (VAS). A score of 100 represents worst pain. Values are mean and standard error. ${ }^{*} p<0.05$ vs. baseline 


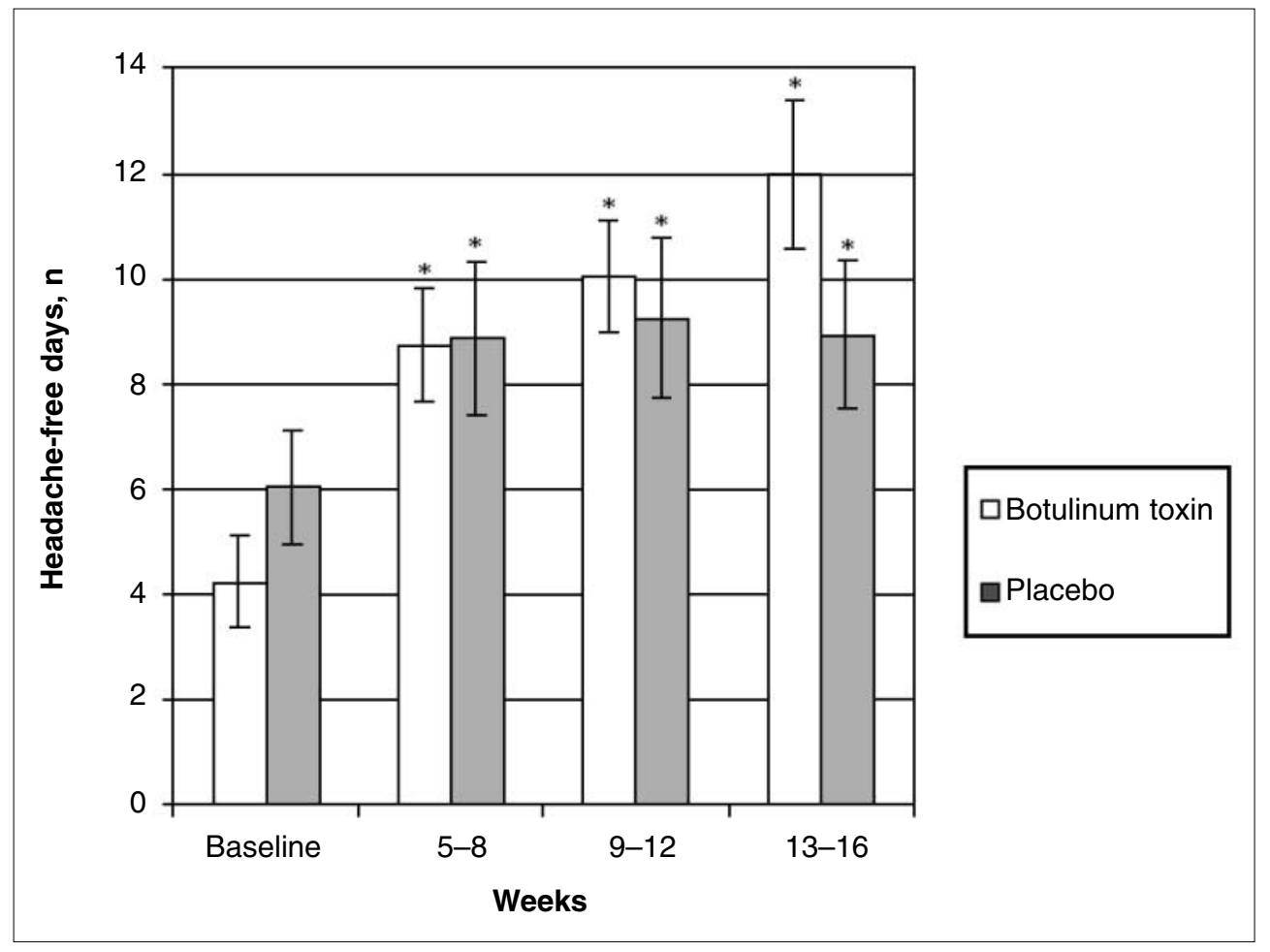

Fig. 2 Number of headache-free days per 4-week period. Values are mean and standard error. $* p=0.005$ vs. baseline

Fig. 3 Number of headache hours per day. Values are mean and standard error. ${ }^{*} p<0.005$ vs. baseline

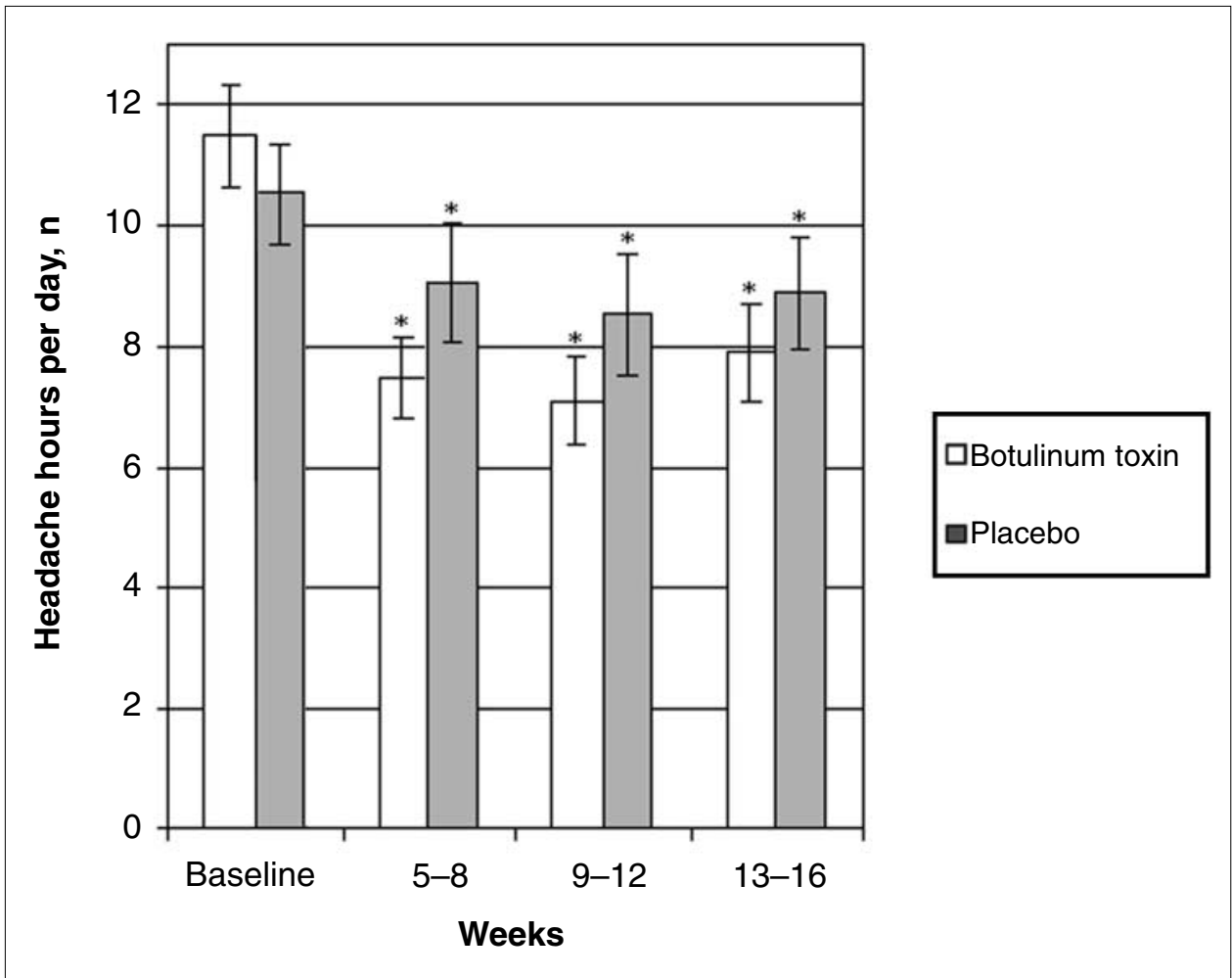

There was no statistically significant change in analgesic intake per day during the follow-up period in either group. However, the BTX-A group showed a trend reduction in the number of headache hours per day was seen in the BTX-A group compared to the placebo group $(p<0.159)$ (Fig. 3). 


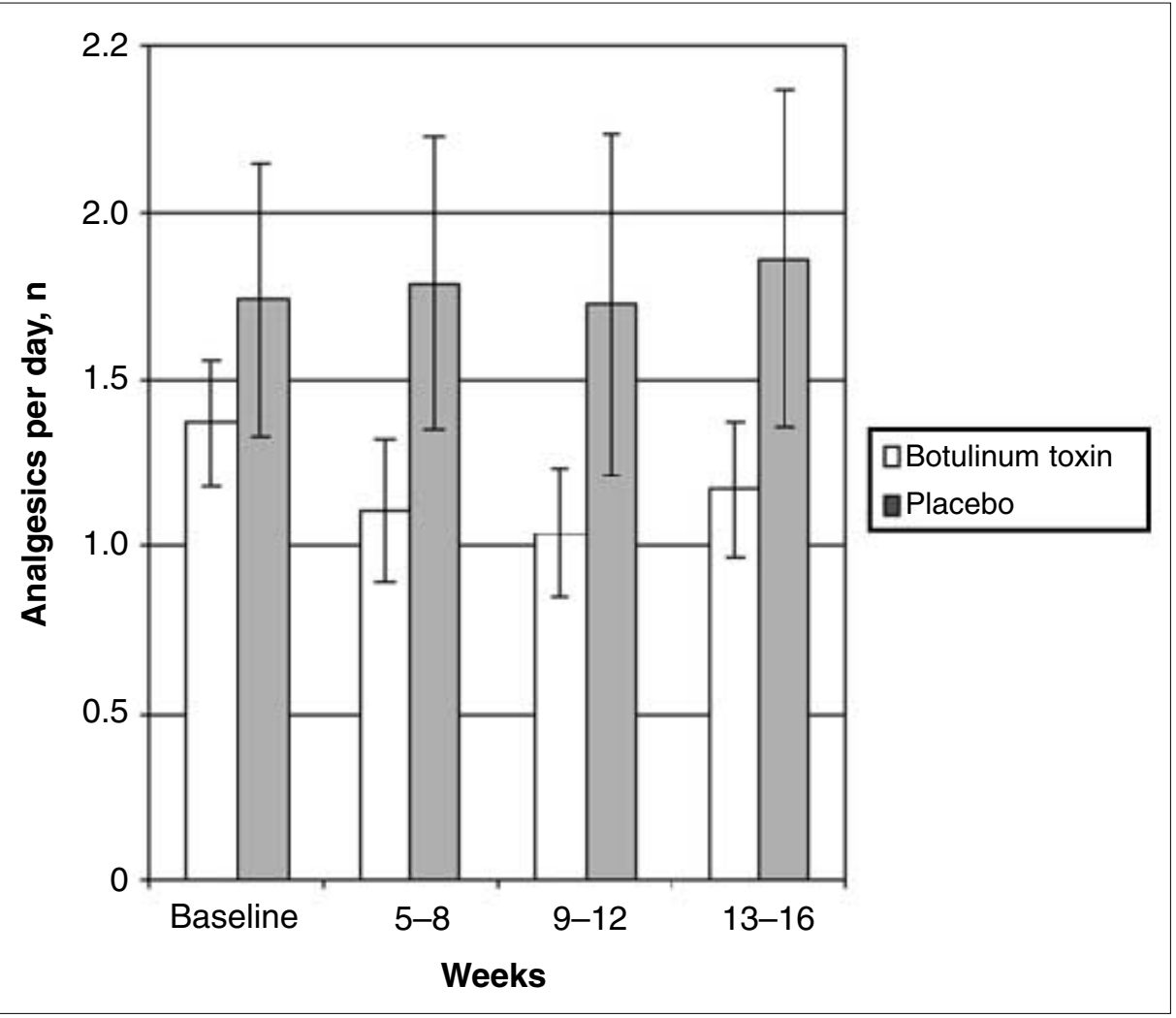

Fig. 4 Number of analgesics taken per day. Values are mean and standard error. Changes vs. baseline are not significant

Fig. 5 Pressure pain thresholds at trigger points, as scored on a 4point scale $(1$, no local tenderness to pressure; 4 , pronounced local tenderness with pain radiation). Values are mean and standard error for the 6 most painful trigger points

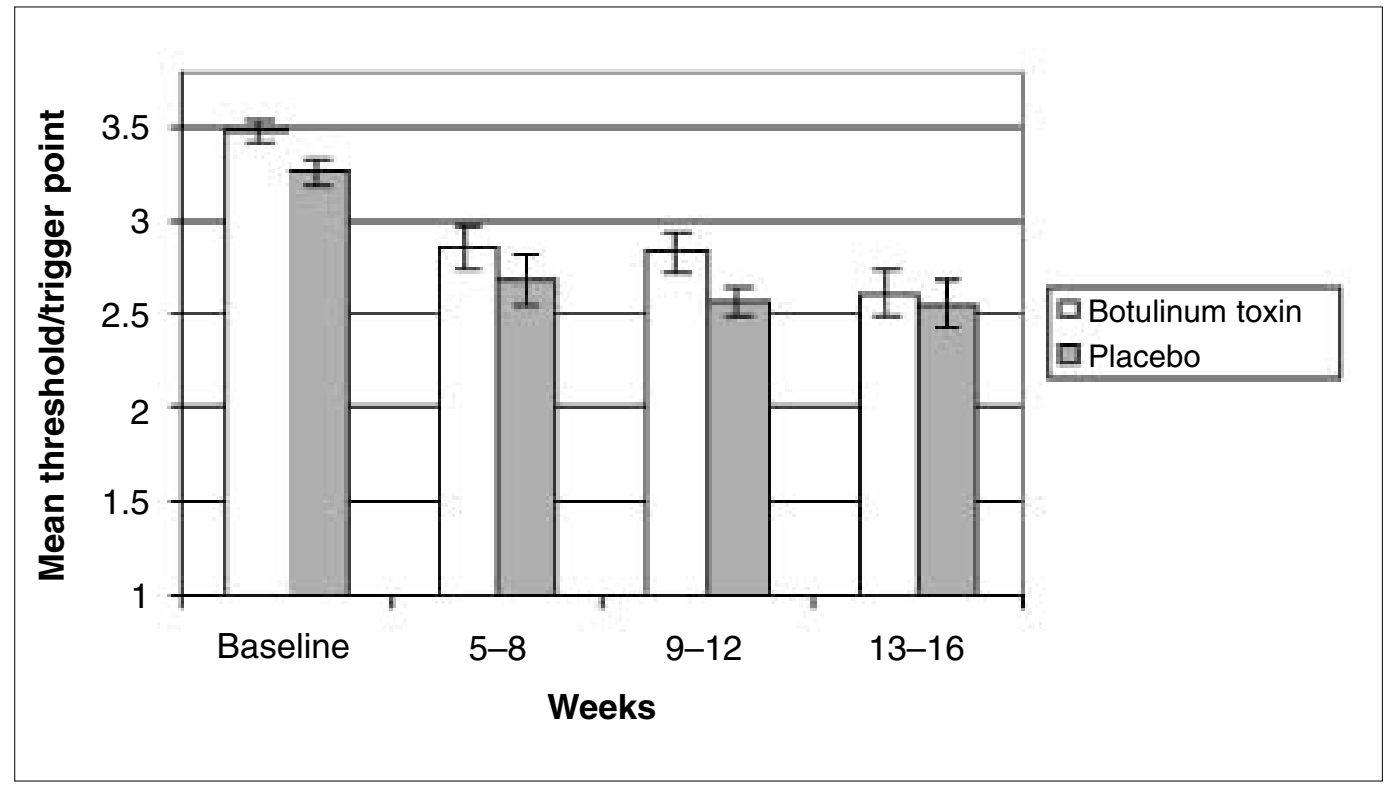

towards reduced analgesic intake over time (weeks 5-8, $p<0.092$; weeks 9-12, $p<0.168$; weeks 13-16, $p<0.108)$ (Fig. 4).

The number of patients who needed to be treated (NNT) for a reduction in the number of headache-free days of at least $50 \%$ was 7 . The NNT for a reduction in the number of headache hours per day of at least $50 \%$ was 6 . However, the NNT for a $50 \%$ reduction in VAS was 28.

The pressure pain thresholds had comparable mean baseline values for both groups and showed no statistically sig- 
nificant changes over time (Fig. 5). The saggital range of motion and biofeedback measurements showed no significant changes either (data not shown).

There were no reports of subjective weakness in the shoulder girdle or neck muscles and none of the patients experienced dysphagia. In the treatment group one patient noted mild local pain at the injection site for three days postinjection.

\section{Discussion}

In 33 patients with $\mathrm{CH}$, treatment with physical therapy and BTX-A or placebo injections reduced headache severity, headache days per month and headache hours per day. There was also a trend towards a greater increase in the number of headache-free days and headache hours per day in the BTX-A treatment group compared to the placebo group. There was no significant change in analgesic intake per day over time. However, when the groups were compared, a trend towards lower analgesic consumption was observed in the BTX-A group. No serious side effects were observed and, in particular, no weakness in the injected muscles or dysphagia was reported. BTX-A showed very good compatibility and did not interact with any other drugs.

Treatment of $\mathrm{CH}$ is usually empirical and includes nonsteroidal anti-inflammatory drugs, muscle relaxants, antidepressants, nerve or root blockades with local anaesthetics, orthopaedic treatment approaches and various forms of physical therapy [12]. In daily practice most patients report partial pain relief after physical therapy, although there have been no controlled studies on the use of physical therapy in the treatment of $\mathrm{CH}$. Our study suggests benefit from physical therapy in $\mathrm{CH}$ patients, although an additional placebo effect cannot be excluded with this study design. Furthermore our study showed that adjunctive BTX-A injections may increase the effect of physical therapy, although no statistically significant improvement could be seen. The lack of statistical significance might be explained by the small number of subjects included, by the fact that the majority of patients were severely affected and by the good treatment effect of physical therapy. However, the NNT for a reduction of at least $50 \%$ in the number of headache-free days was 7 and for a reduction of at least $50 \%$ in the number of headache hours per day, 6 . This indi- cates that a subgroup of patients shows a good response to BTX-A compared to the use of physical therapy alone.

At the end of the trial, BTX-A injections were offered to all 33 patients. Eighteen patients (9 from the treatment group, 9 from the placebo group) were retreated. Five patients ( 3 from the treatment group, 2 from the placebo one) were not retreated because their headaches were only mild. Ten patients were not interested in further injections. Of the retreated patients, 6 did not respond at all and 12 showed a good response ( $>50 \%$ reduction in the number of headache-free days). The duration of the effect was approximately 3.5 months. Recurrent headaches were noted as being less severe than those prior to the first BTX-A treatment. Four patients have so far been retreated up to 4 times. Interestingly, among the 18 patients who underwent retreatment (in open design), 4 of those who had been initially treated with BTX-A did not experience a beneficial effect until the second injection. This is in accordance with the work of Wheeler et al. [2], who described better results after repeated injections of BTX-A.

The role of muscular components in the aetiology of various headache syndromes is not completely known, although all forms of common headache disorders may be influenced or triggered by various myogenic factors. Abnormal sensory inputs due to muscular dysfunction following pathological posture, minor trauma, physical or mental stress might influence central pain-processing pathways. Mild general relaxation of affected head and neck muscles may alter sensory input and interrupt abnormal pain processing. Currently used treatment strategies such as biofeedback, muscle relaxants, physiotherapy and other physical measures are used to reduce muscular tension in headache management. The dosedependent and long-lasting effect of BTX-A could easily be combined with other muscle relaxation strategies to increase and prolong treatment effects.

In conclusion, the combined use of physical measures and adjunctive intramuscular injections of botulinum toxin type A is safe. Adjunctive BTX-A injections seem to further improve cervical headache-related pain. Repeated BTX-A treatments probably show a more marked improvement compared to physical therapy alone. These results warrant further studies including larger numbers of patients who receive physical therapy and adjunctive, repeated BTX-A treatment cycles.

Acknowledgment The study has been supported by a grant of Österreichische National Bank, nr. 7307. 


\section{References}

1. Jankovic J, Brin MF (1999)

Therapeutic uses of botulinum toxin. $\mathrm{N}$ Engl J Med 324:1186-1194

2. Wheeler AH, Goolkasian P, Gretz SS, Fanciullo GJ (1998) A randomized, double-blind, prospective pilot study of botulinum toxin injection for refractory, unilateral, cervicothoracic, paraspinal, myofascial pain syndrome. Spine 23:1662-1667

3. Cheshire WP, Abashian SW, Mann JD (1994) Botulinum toxin in the treatment of myofascial pain syndrome. Pain 59:65-69

4. Freund BJ, Schwartz M (2000)

Treatment of chronic cervical-associated headache with botulinum toxin A: a pilot study. Headache 40:231-236

5. Porta M (2000) A comparative trial of botulinum toxin type A and methylprednisolone for the treatment of myofascial pain syndrome and pain from chronic muscle spasm. Pain 85:101-105

6. Silberstein O, Matthew N, Saper J, Jenkins S (2000) Botulinum toxin type A as a migraine preventive treatment: for the BOTOX Migraine Clinical Research Group. Headache 40:445-450
7. Smuts JA, Baker MK, Smuts HM, Stassen JMR, Rossouw E, Barnard PWA (1999) Prophylactic treatment of chronic tension-type headache using botulinum toxin type A. Eur J Neurol 6[Suppl 4]:99-102

8. Hobson DE, Gladish DF (1997)

Botulinum toxin injection for cervicogenic headache. Headache 37 : 253-255

9. Relja M (1997) Treatment of tensiontype headache by local injection of botulinum toxin. Eur J Neurol 4:S71-S73

10. - (1998) Classification and diagnostic criteria for headache disorders, cranial neuralgias and facial pain. Headache Classification Committee of the International Headache Society. Cephalalgia 8[Suppl 7]:1-96

11. Pearce JM (1995) Cervicogenic headache: a personal view. Cephalalgia 15:463-469

12. Pöllmann W, Keidel M, Pfaffenrath V (1997) Headache and the cervical spine: a critical review. Cephalalgia 17:801-816
13. Pfaffenrath V, Kaube H (1990)

Diagnostics of cervicogenic headache. Funct Neurol 5:159-164

14. Nilsson N (1995) The prevalence of cervicogenic headache in a random population sample of 20-59-year olds. Spine 20:1884-1888

15. Sjaastad O, Fredriksen TA, Pfaffenrath V (1990) Cervicogenic headache: diagnostic criteria. Headache 30:725-726

16. Göbel H, Edmeads JG (2000) Disorders of the skull and cervical spine. In: Olesen J, Tfelt Hansen P, Welch KMA (eds) The headaches, 2nd edn. Lippincott Williams Wilkins, Philadelphia, pp 891-899

17. Aoki KR (2001) Pharmacology and immunology of botulinum toxin serotypes. J Neurol 248[Suppl $1]: \mathrm{I} / 3-\mathrm{I} / 10$

18. Bellometi S, Galzigna L (1998) Serum levels of prostaglandin and leukotriene after thermal mud pack therapy. J Investig Med 46(4):140-145 